\title{
Quantitative image analysis as a tool for Yarrowia lipolytica dimorphic growth evaluation in different culture media
}

\author{
A. Braga ${ }^{a, 1}$, D.P. Mesquita ${ }^{a, 1}$, A.L. Amaral ${ }^{a, b}$, E.C. Ferreira ${ }^{a}$, I. Belo $^{a, *}$ \\ a CEB-Centre of Biological Engineering, University of Minho, Campus de Gualtar, 4710-057 Braga, Portugal \\ ${ }^{\mathrm{b}}$ Instituto Politécnico de Coimbra, ISEC, DEQB, Rua Pedro Nunes, Quinta da Nora, 3030-199 Coimbra, Portugal
}

\section{A R T I C L E I N F O}

\section{Article history:}

Received 14 July 2015

Received in revised form 27 October 2015

Accepted 30 October 2015

Available online 3 November 2015

\section{Keywords:}

Dimorphic growth

$\gamma$-Decalactone

Y. lipolytica

Quantitative image analysis

\begin{abstract}
A B S T R A C T
Yarrowia lipolytica, a yeast strain with a huge biotechnological potential, capable to produce metabolites such as $\gamma$-decalactone, citric acid, intracellular lipids and enzymes, possesses the ability to change its morphology in response to environmental conditions. In the present study, a quantitative image analysis (QIA) procedure was developed for the identification and quantification of Y. lipolytica W29 and MTLY40-2P strains dimorphic growth, cultivated in batch cultures on hydrophilic (glucose and $N$-acetylglucosamine (GlcNAc) and hydrophobic (olive oil and castor oil) media. The morphological characterization of yeast cells by QIA techniques revealed that hydrophobic carbon sources, namely castor oil, should be preferred for both strains growth in the yeast single cell morphotype. On the other hand, hydrophilic sugars, namely glucose and GlcNAc caused a dimorphic transition growth towards the hyphae morphotype. Experiments for $\gamma$-decalactone production with MTLY40-2P strain in two distinct morphotypes (yeast single cells and hyphae cells) were also performed. The obtained results showed the adequacy of the proposed morphology monitoring tool in relation to each morphotype on the aroma production ability. The present work allowed establishing that QIA techniques can be a valuable tool for the identification of the best culture conditions for industrial processes implementation.
\end{abstract}

(c) 2015 Elsevier B.V. All rights reserved.

\section{Introduction}

Yarrowia lipolytica is an obligate aerobic non-conventional yeast which has been gaining an unquestionable interest in its exploitation in many biotechnological and environmental applications. This yeast is also a dimorphic microorganism capable to grow in two distinct morphotypes, usually as yeast single cells or as filamentous hyphae, being the two morphotypes reversible. It is believed that the dimorphism of $Y$. lipolytica, as well as other species, provides a mechanism for responding to adverse conditions (Kawasse et al., 2003). Naturally, Y. lipolytica grows as a mixture of yeasts and short mycelial cells. Nevertheless, it can grow as a quasihomogeneous population of either yeast-like cells or hyphae, under controlled conditions (Guevara-Olvera et al., 1993; Rodríguez and Domıínguez, 1984; Ruiz-Herrera and Sentandreu, 2002). The role of different factors has been previously described in dimorphic transition of $Y$. lipolytica, such as carbon and nitrogen sources (Rodrí́guez and Domiínguez, 1984; Ruiz-Herrera and Sentandreu,

\footnotetext{
* Corresponding author. Fax: +35 1253604429.

E-mail address: ibelo@deb.uminho.pt (I. Belo).

1 These authors contributed equally to this work.
}

2002; Szabo and Stofanikova, 2002) and medium pH (Ruiz-Herrera and Sentandreu, 2002). Indeed, Y. lipolytica transition from yeast to hyphae morphotype was detected in the presence of casein, olive oil, $\mathrm{N}$-acetylglucosamine, citrate and serum (Guevara-Olvera et al. 1993; Kawasse et al., 2003; Kim et al., 2000; Pérez-Campo and Domínguez, 2001; Ruiz-Herrera and Sentandreu, 2002; Ota et al., 1984; Zinjarde et al., 1998). However, the critical factor affecting the transition of Y. lipolytica from single cells to hyphae, independently of the nature of carbon and/or nitrogen source used, seems to be the dissolved oxygen concentration. Under highly aerated conditions, Y. lipolytica cultures consisted of single cells, by contrast low aeration conditions induced hyphae forms (Bellou et al., 2014). Furthermore, also environmental conditions affect the dimorphism in Y. lipolytica such as the medium $\mathrm{pH}$, as it has been reported that hyphae formation is maximal at $\mathrm{pH}$ near neutrality and decreases as $\mathrm{pH}$ lowers to become almost null at $\mathrm{pH} 3$, with citrate as an important positive effector of hyphae formation (Ruiz-Herrera and Sentandreu, 2002). An important progress has been made recently, with the identification of the genes playing a crucial role in $Y$. lipolytica dimorphism: genes involved in the mitogen-activated protein kinase and protein kinase A pathways, Rho family, among others (Cervantes-Chávez et al., 2009; Hurtado and Rachubinski, 1999; Martinez-Vazquez et al., 2013; Morales-Vargas et al., 2012). 
The morphogenetic shift of $Y$. lipolytica has a practical importance when biotechnological applications are designed. In fact, this wide morphological change greatly affects fermentation behavior, since it induces rheological changes and consequently leads to mass and heat transfer changes in the bioreactor (Coelho et al., 2010). It is known that $Y$. lipolytica is extensively used for enzyme production, lipids accumulation and aromatic compounds production, although the processes productivity is strongly affected by the cells morphology (Coelho et al., 2004).

In the last few years, microscopic examination has been ever more useful for determining the biomass structure, composition, and contents. Coupled to microscopy, the technological evolution and advances in digital image acquisition and computer processing capabilities allow for a fast and efficient characterization of the process biomass. Regarding yeasts characterization, solely a limited number of works can be found in the literature using quantitative image analysis (QIA). Coelho et al. (2004, 2007) developed automatic QIA procedures for Saccharomyces cerevisiae allowing the discrimination between budding and single cells, as well as the determination of cell size distribution under different experimental conditions. These results showed the potential of QIA to study the physiological state assessment and cell division analyses of yeast cell cultures used in industrial processes. Braga et al. (2015a) also used QIA to study the influence of mechanical and pneumatic agitation in the $Y$. lipolytica morphology, during $\gamma$-decalactone production in a stirred tank reactor (STR) and in an airlift bioreactor. However, up to the present, QIA has never been applied for characterizing dimorphic growth and/or transition of $Y$. lipolytica in different carbon sources.

This research is mainly focused on the development of a QIA procedure for the automatic identification and quantification of yeast (oval) single cells and (filamentous) hyphae cells of Y. lipolytica due to dimorphic transition using $\mathrm{N}$-acetylglucosamine, glucose, castor oil, and olive oil as carbon sources. Finally, the obtained image analysis data was used to identify the best culture conditions for industrial processes implementation.

\section{Experimental procedures}

\subsection{Microorganism}

The strains used in this work were $Y$. lipolytica wild type (WT) strain W29 (ATCC20460) (Barth and Gaillardin, 1996) and its derived mutant with modifications in the lipid metabolism at the peroxisomal $\beta$-oxidation pathway (deleted for acyl-CoA oxidase encoding genes and overexpressing POX2) - MTLY40-2P strain ( $\triangle$ pox2 $\triangle$ pox3 $\triangle$ pox4 $\triangle$ pox5+ pPOX2-POX2), that was proven to produce high levels of $\gamma$-decalactone from castor oil (Braga et al., 2015b; Groguenin et al., 2004).

\subsection{Dimorphic transition}

Dimorphic transition was achieved essentially as described by Guevara-Olvera et al. (1993). Cells were cultured for $48 \mathrm{~h}$ on YPDA medium (agar $30 \mathrm{~g} \mathrm{~L}^{-1}$, glucose $20 \mathrm{~g} \mathrm{~L}^{-1}$, peptone $20 \mathrm{~g} \mathrm{~L}^{-1}$, yeast extract $10 \mathrm{~g} \mathrm{~L}^{-1}$ ) at $27^{\circ} \mathrm{C}$ to inoculate (cell density of $0.5 \mathrm{~g} \mathrm{~L}^{-1}$ ) a $500 \mathrm{~mL}$ baffled Erlenmeyer flask containing $200 \mathrm{~mL}$ of glucose medium (YPD medium: glucose $20 \mathrm{~g} \mathrm{~L}^{-1}$, peptone $20 \mathrm{~g} \mathrm{~L}^{-1}$, yeast extract $\left.10 \mathrm{~g} \mathrm{~L}^{-1}\right)$. Cultures were incubated overnight at $27^{\circ} \mathrm{C}$, $140 \mathrm{rpm}$. Cells were centrifuged, re-suspended in the same volume of sterile distilled water, agitated for $3 \mathrm{~h}$ at $27^{\circ} \mathrm{C}$, centrifuged, washed once with sterile distilled water, and re-suspended in a small volume of sterile distilled water. Cell suspensions were stored at $4{ }^{\circ} \mathrm{C}$ overnight and further used as inoculum in experiments to study the dimorphic transition of Y. lipolytica. Cells kept at $0{ }^{\circ} \mathrm{C}$ in an ice bath were inoculated in $100 \mathrm{~mL}$ of medium (ca. $1 \mathrm{~g} \mathrm{~L}^{-1}$ ), dispensed in $250 \mathrm{~mL}$ Erlenmeyer flasks, and maintained at $37-38^{\circ} \mathrm{C}$. For the dimorphic transition YNB medium $(0.67 \%$ YNB (yeast nitrogen base) without amino acids/ammonium sulfate, $0.5 \%$ ammonium sulfate, $1 \%$ carbon source and $100 \mathrm{mM}$ citrate buffer $\mathrm{pH}$ 7) was used. After 10-15 min, the Erlenmeyer flasks were incubated at $27^{\circ} \mathrm{C}, 170 \mathrm{rpm}$ for about $12 \mathrm{~h}$, the optimal period for hyphae formation (Guevara-Olvera et al., 1993).

\subsubsection{Effect of different carbon sources on dimorphic transition}

Previously, it was described that hyphae formation was obtained when GlcNAc was used as carbon source (Guevara-Olvera et al., 1993; Rodríiguez and Domínguez, 1984). Therefore, we compared hyphae formation in hydrophilic (glucose and GlcNAc) and hydrophobic (olive oil and castor oil) substrates. In the experiments with hydrophobic substrates, the medium was emulsified with $0.1 \%$ Triton X and YPD medium ( $\mathrm{pH}$ 6.5, without citrate buffer) was used as control.

\subsection{Samples preparation for image acquisition}

In the dimorphic transition experiments, three biological replicates were prepared for each condition. From each cultured medium, a $1 \mathrm{~mL}$ sample was collected every two hours until $12 \mathrm{~h}$, for the implementation of a QIA procedure in bright-field microscopy. These samples were further centrifuged at $5000 \mathrm{rpm}$ for $5 \mathrm{~min}$, the supernatant was discarded and the pellet cells were washed, resuspended with distilled water and centrifuged using the same conditions. This procedure was repeated three times in order to remove the lipidic material (Braga et al., 2015a). The pellet cells were then re-suspended in $1 \mathrm{~mL}$ of distilled water and then visualized in bright-field microscopy.

\subsubsection{Bright-field image acquisition}

A recalibrated micropipette with a sectioned tip at the end, with a large enough diameter to allow larger cells and cell aggregates to flow, was used to deposit samples on slides. Three slides per sample were used, and for each slide a volume of $10 \mu \mathrm{L}$ was covered with a $20 \mathrm{~mm} \times 20 \mathrm{~mm}$ cover slip for visualization and image acquisition. Images were acquired in the upper, middle, and bottom of the slide resulting in a total of 150 images $(3 \times 50$ images/slide). The slides were examined by means of an Olympus BX51 optical microscope (Olympus, Tokyo, Japan), at $100 \times$ total magnification, coupled with an Olympus DP72 camera (Olympus, Tokyo, Japan). Images were acquired at $1360 \times 1024$ pixels and 8-bit format through the commercial software CellB (Olympus, Tokyo, Japan).

\subsubsection{Bright-field image processing and analysis}

The yeast and hyphae morphological descriptors were determined through the use of image processing and analysis programs developed in Matlab 7.3 (The Mathworks, Inc., Natick) language. The main stages of the image processing program comprise the image pre-processing, segmentation, hyphae and yeast cells recognition, and image post processing steps. Finally, the yeast and hyphae cells binary images were saved and later used for their contents and size characterization in the QIA parameters determination step as described below.

A more detailed description of the image processing methods is next presented.

\subsection{Image pre-processing}

The image pre-processing stage depends on the enhancement of the grayscale images by background determination and background removal. In this stage, the original image is first divided by a background image to minimize background light differences. The 


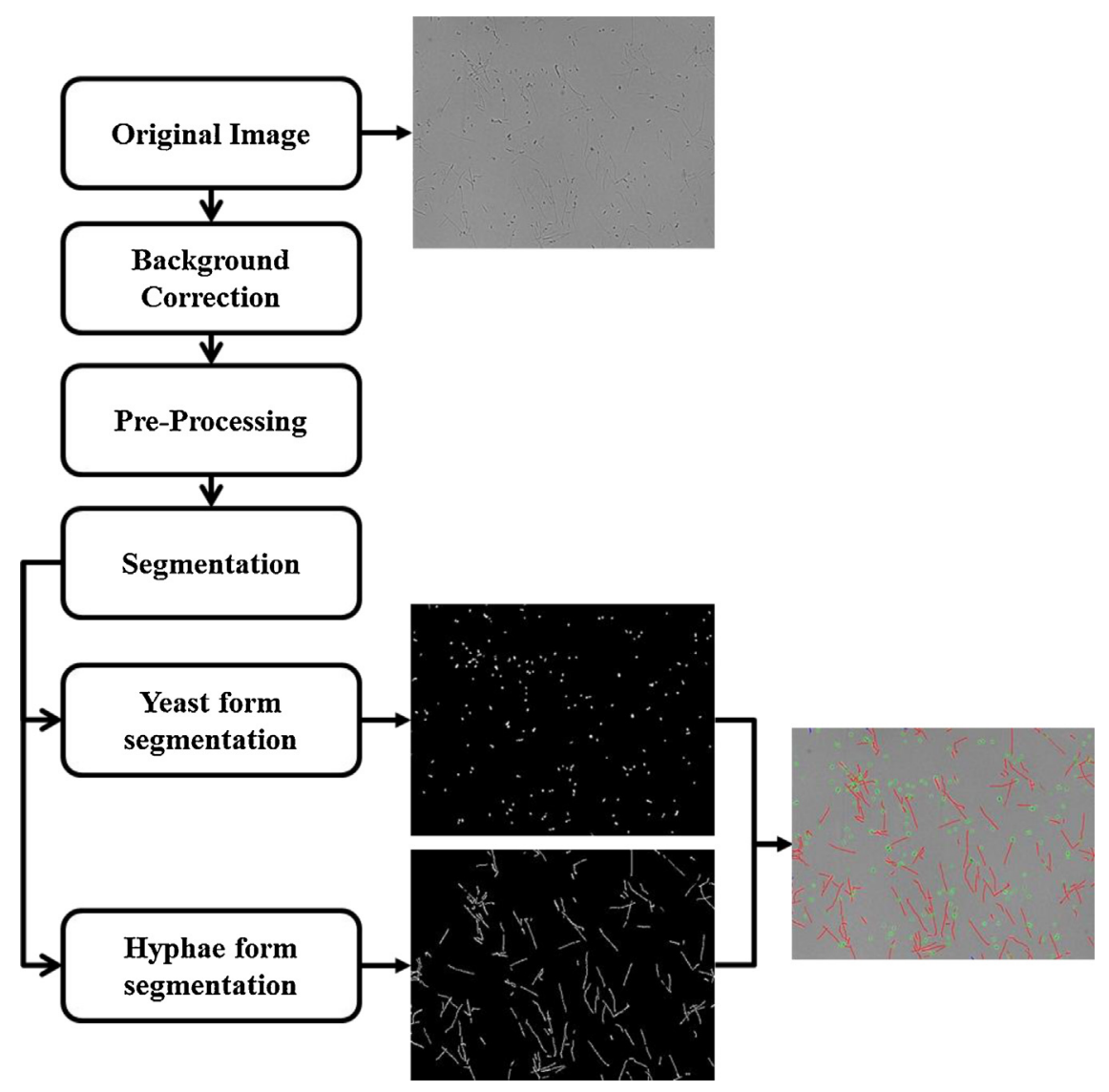

Fig. 1. Schematic representation of the QIA methodology.

resulting background corrected image is further enhanced by using a series of closing and opening morphological operators in order to improve the contrast of the cells boundaries.

\subsubsection{Segmentation}

This stage consists primarily on the yeast and hyphae morphotype recognition through segmentation by a predefined 0.9 threshold value. Then the resulting binary image is processed in order to fill small holes ( 6 pixels, or approximately $4.5 \mu \mathrm{m}$, in diameter) in the interior of the cells. A native Matlab function is used for that purpose.

\subsubsection{Hyphae and yeast cells recognition}

Next, the hyphae morphotype is determined taking into account a combined width and morphology approach. In that sense, the objects presenting a width bellow $9 \mu \mathrm{m}$ are recognized by a series of erosion and dilation morphological operations. From these, the objects presenting a gyration radius (Pons and Vivier, 1998) above 1 are considered as hyphae cells. The remaining objects, if above $3.5 \mu \mathrm{m}$ in diameter, are considered as yeast cells or cell aggregates.

\subsubsection{Image post processing}

Finally, all the objects cut off by the image boundaries are removed by a native Matlab function and the final yeasts and hyphae cells binary images are saved (Fig. 1).

\subsubsection{QIA parameters determination}

Following the image processing step, the yeasts and hyphae cells binary images were analyzed in order to characterize both morphotypes in terms of their contents and most relevant size parameters. In all cases, a calibration factor of $0.7383 \mu \mathrm{m} \mathrm{pixel}^{-1}$ (determined by the use of a micrometer slide) was employed. The studied parameters are further described below.

The total yeast area per volume (TY/Vol) and total hyphae length per volume ( $\mathrm{TH} / \mathrm{Vol})$ were determined for each replicate as the sum of all yeast areas per volume and the sum of all hyphae lengths per volume, respectively. The total yeast area (TY) represents the projected area of all yeast cells onto the image plane, whereas the total hyphae length $(\mathrm{TH})$ was determined by a QIA morphological thinning procedure to a 1 pixel width of all hyphae cells. Upon the determination of TY in $\mu \mathrm{m}^{2}$ and $\mathrm{TH}$ in $\mu \mathrm{m}$, by the use of the calibration factor, these values were divided by the corresponding volume of each image. For each replicate a total of 150 images (50 images $\times 3$ slides) was used. Finally, for each sample, the average value of the 3 replicates was then determined. Furthermore, the total hyphae length per total yeast area ratio (TH/TY) was also determined.

\subsection{Biotransformation experiments}

In order to evince the importance of cell morphology monitoring in bioprocesses, $\gamma$-decalactone production by $Y$. lipolytica in two different morphotypes (yeast or hyphae cells) was evaluated. MTLY40-2P strain was employed since it was proven to produce high levels of $\gamma$-decalactone from castor oil and methyl ricinoleate, due to severely decreased short-chain fatty acid degradation and enhanced long-chain fatty-acid $\beta$-oxidation through the overexpression of Aox2p (Braga et al., 2015b; Groguenin et al., 2004). For this purpose, two different experiments were conducted. In the first experiment cells were employed without previous dimorphic induction. Cells from YPDA medium were used to inoculate a pre-growth culture ( $200 \mathrm{~mL}$ of YPD medium) with cell den- 


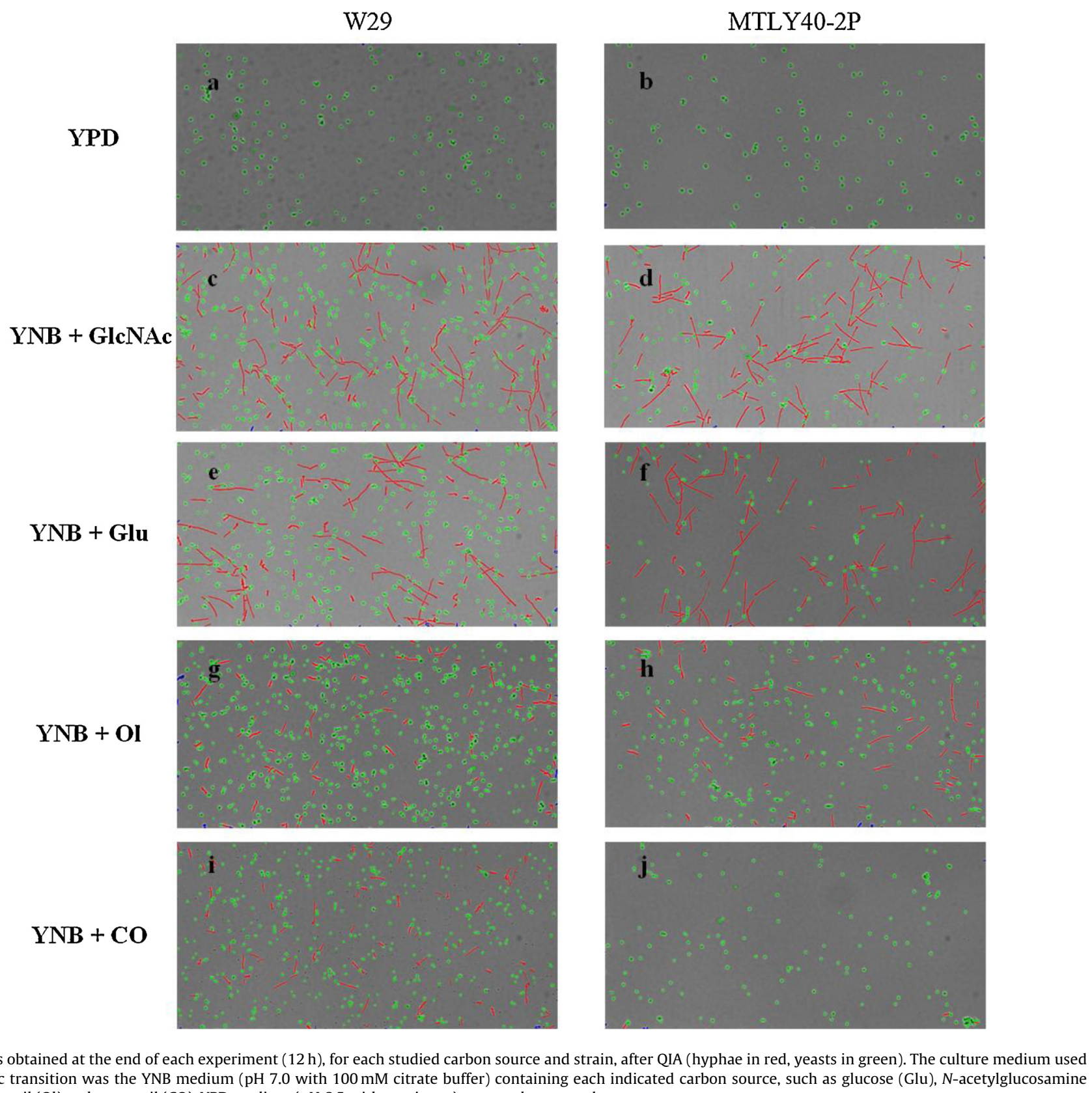

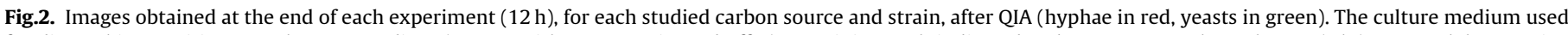

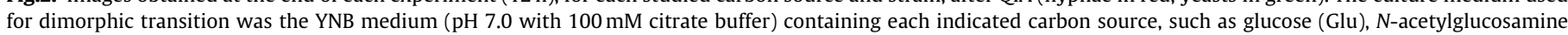
(GlcNAc), olive oil (Ol) and castor oil (CO). YPD medium (pH 6.5 without citrate) was used as control.

sity of $0.5 \mathrm{~g} \mathrm{~L}^{-1}$, incubated overnight at $27^{\circ} \mathrm{C}, 140 \mathrm{rpm}$. Cells were then centrifuged and used to inoculate $200 \mathrm{~mL}$ of the biotransformation medium ( $30 \mathrm{~g} \mathrm{~L}^{-1}$ castor oil, $6.7 \mathrm{~g} \mathrm{~L}^{-1} \mathrm{YNB}$ with amino acids, $2.5 \mathrm{~g} \mathrm{~L}^{-1} \mathrm{NH}_{4} \mathrm{Cl}$ and $3 \mathrm{~g} \mathrm{~L}^{-1}$ Tween 80 ) with cell density of $0.5 \mathrm{~g} \mathrm{~L}^{-1}$. In the second experiment, after selecting the best conditions for hyphae induction (medium with glucose), dimorphic transition was induced as previously described in Section 2.2. Then, the cells were centrifuged and used to inoculate $200 \mathrm{~mL}$ of the biotransformation medium with cell density of $0.5 \mathrm{~g} \mathrm{~L}^{-1}$. Finally, samples were collected over time for $\gamma$-decalactone quantification, cell concentration and substrate consumption. $\gamma$-Decalactone was extracted from $2 \mathrm{~mL}$ samples with $2 \mathrm{~mL}$ diethyl ether and the organic phase was analyzed by gas chromatography (GC) as previously described by Braga et al. (2015a). Castor oil consumption was determined by fatty acids methyl esters (FAME) analysis by GC (Braga, 2014).

\section{Results and discussion}

\subsection{Yeast-to-hyphae transition by QIA}

QIA is a powerful tool for microorganisms' characterization. Amongst other applications, QIA has been used to relate fermentation broth rheology to morphology (Cox et al., 1998), and study the impact of bioreactor agitation (mechanical or pneumatic) in yeast cells morphology (Braga et al., 2015a). However, QIA studies for morphological analysis of dimorphism microorganisms are still lacking.

The automatic identification and quantification of yeast (oval) single cells and (filamentous) hyphae cells of Y. lipolytica caused by the presence of different inducers in culture medium (YNB medium) was sought using QIA. The growth of Y. lipolytica strains showed a differential dimorphic behavior in response to the 

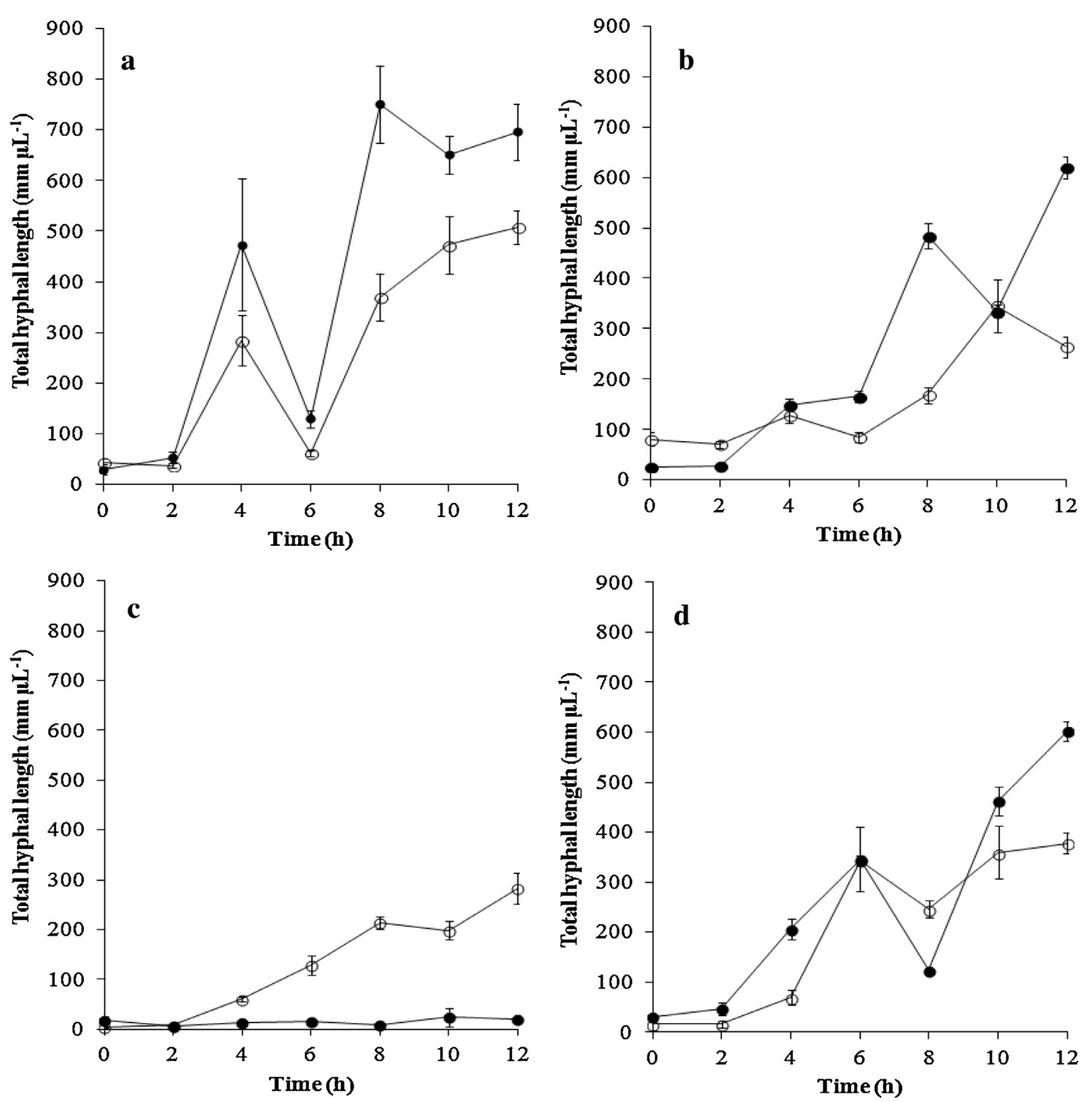

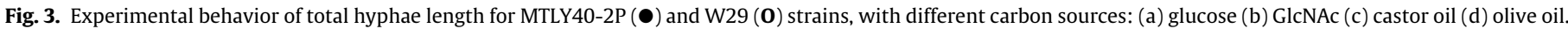
Data are presented as average and standard deviation of three independent experiments.

presence of hydrophobic (olive oil and castor oil) or hydrophilic (GlcNAc and glucose) carbon sources (Fig. 2).

It has been observed that the capability to grow in hyphae morphotype is variable among different strains of Y. lipolytica (Barth and Gaillardin, 1997). During the present research, the same conclusion could be achieved over the global comparison between MTLY40-2P strain and the parental strain W29, as Fig. 2 demonstrates.

Regarding the final labeled images obtained by QIA methodology for the YPD medium, the common medium for yeast cells growth and in this work used as control, as shown in Fig. 2, cells were present in the yeast morphotype (in green) throughout the experiments for both strains, indicating that YPD is not able to induce dimorphic transition, as expected.

When both strains were subjected to the presence of hydrophilic sources, such as glucose or GlcNAc, the dimorphic transition of $Y$. lipolytica cells was induced (Fig. 2c-f). This was clearly revealed by the QIA where the hyphae morphotype was identified as hyphae cells (in red). However, there are some discrepancies in the literature concerning the morphology of $Y$. lipolytica on different hydrophilic substrates under varied culture conditions. For instance, the growth of $Y$. lipolytica LGAM S(7) 1 was reported as being restricted to the yeast morphotype with glucose (Papanikolaou et al., 2002), whereas other reports have indicated that hyphae formation is triggered with glucose and other hydrophilic materials (Kim et al., 2000; Perez-Campo and Dominguez, 2001). On the other hand, GlcNAc was also described as a good inducer for dimorphic transition of Y. lipolytica (GuevaraOlvera et al., 1993; Rodríguez and Domínguez, 1984).

Additionally, when hydrophobic substrates were used, it was clearly observed at the end of the experiments that the MTLY40-2P strain and the parental strain $\mathrm{W} 29$ responded in a different manner. When olive oil ( $\mathrm{Ol})$ was used as carbon source, growth was promoted in the hyphae morphotype for both strains (Fig. $2 \mathrm{~g}-\mathrm{h}$ ). On the other hand, with castor oil (CO) a suboptimal hyphae formation was induced (Fig. $2 \mathrm{i}-\mathrm{j}$ ) and higher amounts of the yeast morphotype were present. Comparing the results obtained after QIA analysis it was possible to observe a higher amount of red hyphae cells (in red) in the experiments with Ol contrasting with a predominance of yeast cells (in green) in CO experiments. This could be explained by the fatty acid composition of both oils. It is known that oleic acid 

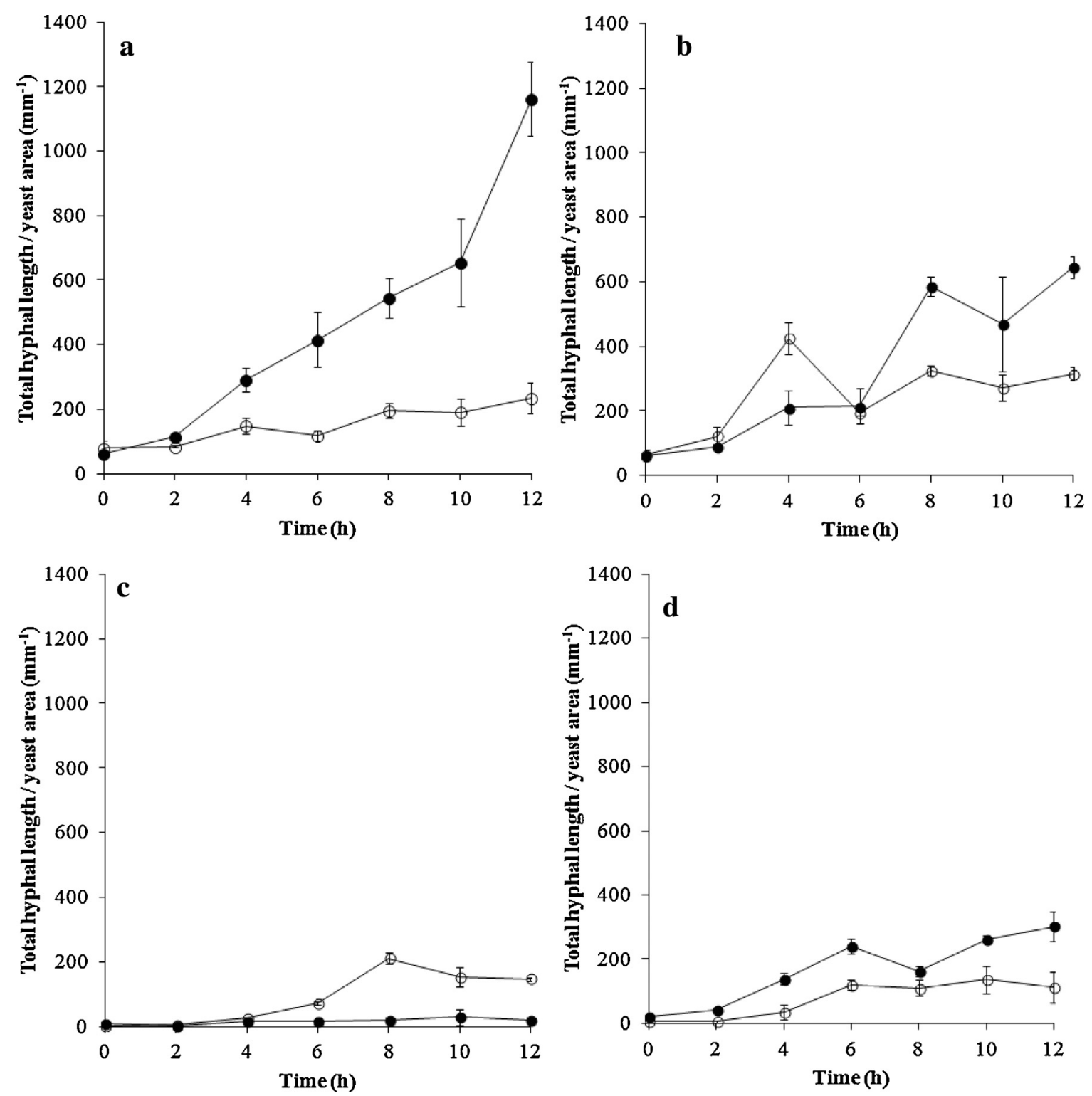

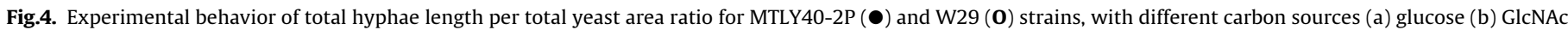
(c) castor oil (d) olive oil. Data are presented as average and standard deviation of three independent experiments.

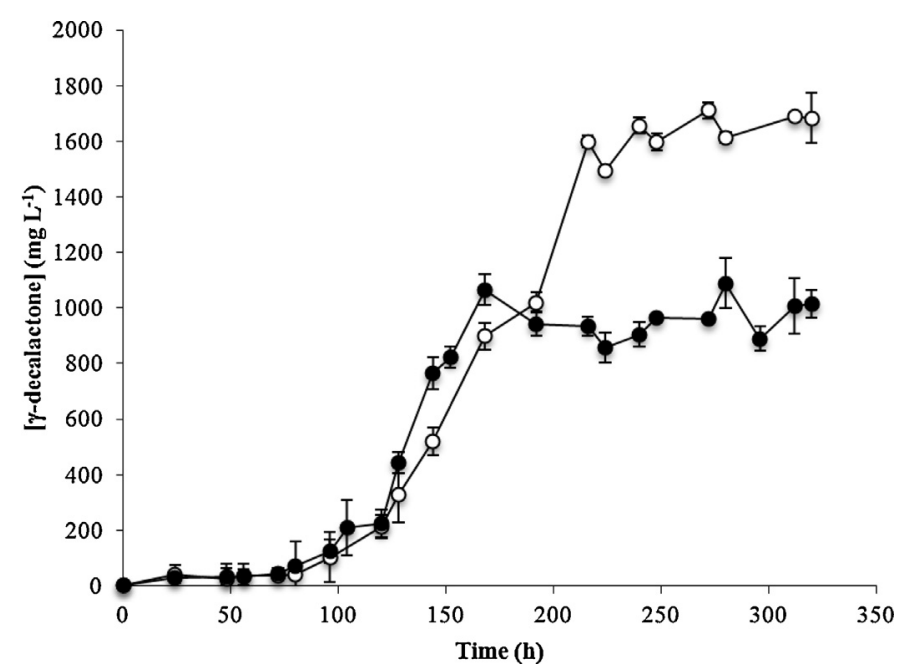

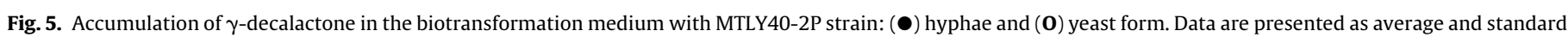
deviation of two independent experiments. 

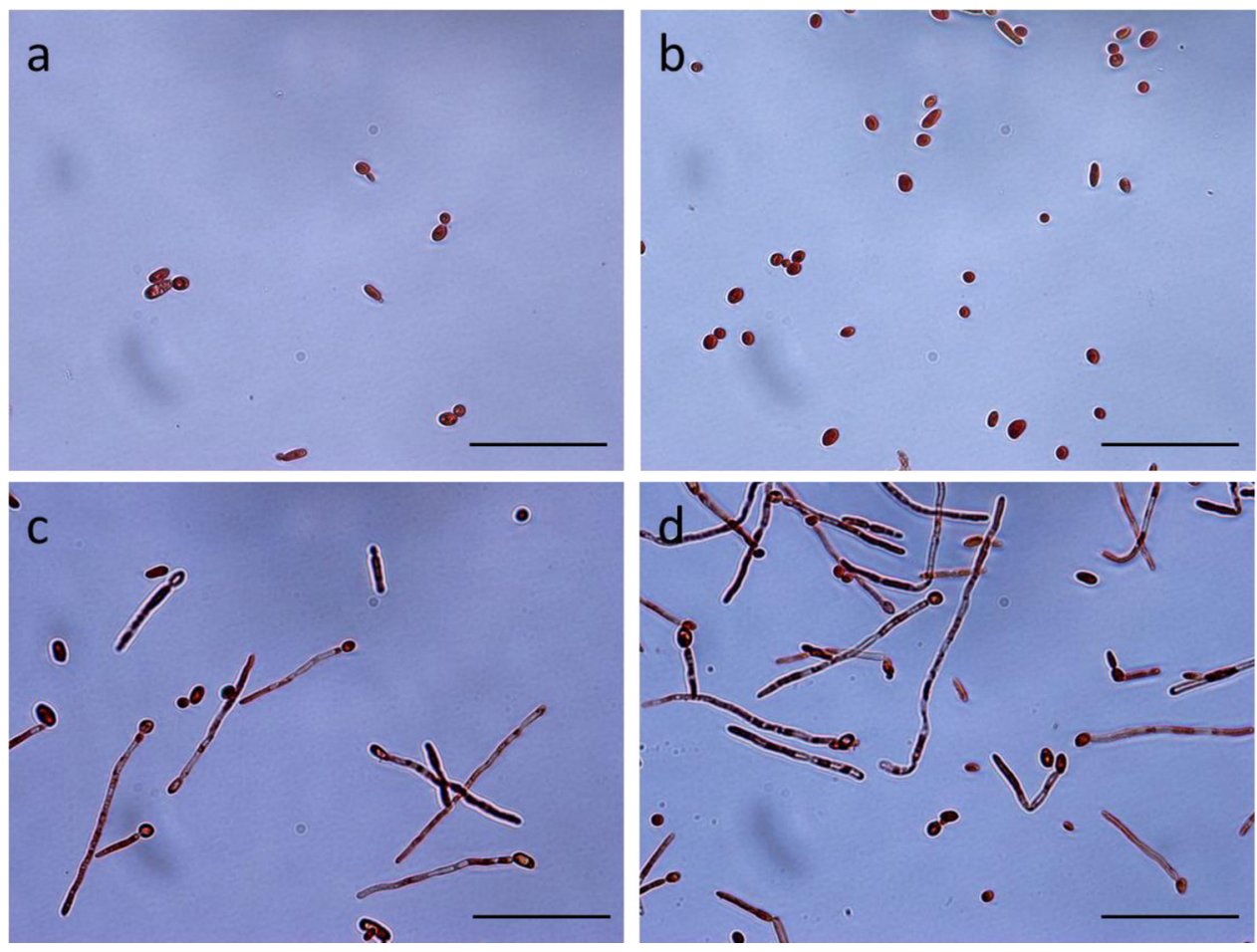

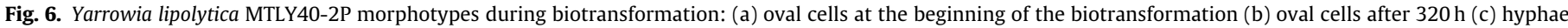
cells at the beginning of the biotransformation (d) hyphae cells after $320 \mathrm{~h}$. The scale bar represents $50 \mu \mathrm{m}$.

is the main fatty acid in $\mathrm{Ol}$ and ricinoleic acid is the main fatty acid in CO (Puthli et al., 2006). Taking into account a literature review, most of previous studies using hydrophobic carbon sources for the transition from yeast to hyphae morphotype in Y. lipolytica, reported solely the use of oleic acid (Ofek et al., 1983; Ota et al., 1984). To the authors' knowledge, castor oil has never been described before as an inducer in studies concerning the dimorphic transition.

The analysis of total hyphae length per volume $(\mathrm{TH} / \mathrm{Vol})$, one of the most significant morphological parameter provided by QIA, showed that hyphae began to appear in about $4 \mathrm{~h}$ after transfer to the tested media with the different inducers (Fig. 3), with the exception of CO for the MTLY40-2P strain. Seemingly, the conversion of the yeast cells into hyphae morphotype is seen to occur from that time onwards, and after $12 \mathrm{~h}$ of induction $\mathrm{TH} / \mathrm{Vol}$ is the highest (in most cases), indicating the largest hyphae morphotype contents.

For both strains, glucose was the best inducer for the dimorphic transition in the experimental conditions analyzed. A TH/Vol

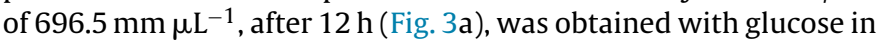
the case of MTLY40-2P. The hyphae formation decreased $11 \%$ when glucose was replaced with GlcNAc to a $\mathrm{TH} / \mathrm{Vol}$ of $619.2 \mathrm{~mm} \mu \mathrm{L}^{-1}$ at the same time. In the case of the parental strain W29, a max-

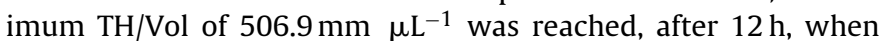

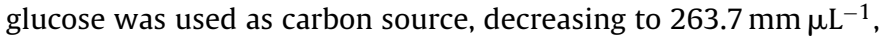
at the same time, when GlcNAc was used as the sole carbon source (Fig 3b). A similar behavior was observed in the experiments with Ol where $\mathrm{TH} / \mathrm{Vol}$ of $601.9 \mathrm{~mm}_{\mu} \mathrm{L}^{-1}$ and $377.7 \mathrm{~mm}_{\mu} \mathrm{L}^{-1}$ where obtained, after $12 \mathrm{~h}$, for MTLY40-2P and W29, respectively (Fig 3d). In the medium containing $\mathrm{CO}$ a slightly lower hyphae induction

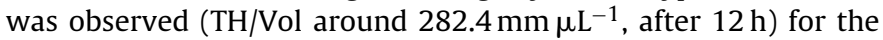
parental strain W29, whereas no significant hyphae induction was observed for MTLY40-2P strain (Fig. 3c).

In the case of $Y$. lipolytica, it has been reported that the morphological shift depends on the nature of the carbon and nitrogen sources, the environmental conditions (temperature, $\mathrm{pH}$, oxygen), and the presence of serum or citrate, although, as previous described, there are discrepancies in literature concerning these topics (Domínguez et al., 2000; Pérez-Campo and Domínguez, 2001). However, it is known that Y. lipolytica normally switches morphology from yeast to hyphae in response to the presence GlcNAc, glucose or Ol in their growth medium (Novotony et al., 1994; Ota et al., 1984; Rodríguez and Domínguez, 1984) as was observed in the present work.

Pons et al. (1993) reported one of first QIA applications on yeast single cell morphotype using a semi-automatic method for the characterization of yeast size and shape. In the present work our QIA methodology was able to automatically identify, characterize and quantify both yeast and hyphae morphological status. The key parameter "Total hyphae length per total yeast area ratio" (TH/TY) was obtained representing quantitatively the morphological cells shift (Fig. 4).

Fig. 4 shows a clear distinction between the hydrophobic and hydrophilic carbon sources, with a higher impact of the first in the dimorphic shift of both strains.

It was observed that with glucose (Fig. 4a) the TH/TY ratio was 1162 and $234.3 \mathrm{~mm}^{-1}$, after $12 \mathrm{~h}$, for MTLY40-2P and W29, respectively. Regarding GlcNAc experiments (Fig. 4b), a TH/TY ratio of $645.1 \mathrm{~mm}^{-1}$ for MTLY40-2P and $315.5 \mathrm{~mm}^{-1}$, after $12 \mathrm{~h}$, for W29 was achieved indicating a morphological shift from yeast to hyphae morphotype in both cases. Lower TH/TY ratios were obtained using hydrophilic carbon sources, showing the lower ability to induce morphological switches (Fig. 4c-d). In the medium containing CO (Fig. 4c), TH/TY ratios of $147.9 \mathrm{~mm}^{-1}$ and $20.1 \mathrm{~mm}^{-1}$, after $12 \mathrm{~h}$, were achieved for W29 and MTLY40-2P, respectively. An opposite behavior was observed in the experiments with Ol (Fig. 4d) where for MTLY40-2P a TH/TY ratio of $302.3 \mathrm{~mm}^{-1}$ was obtained, after $12 \mathrm{~h}$, and for W29 a much lower TH/TY ratio of around $113.2 \mathrm{~mm}^{-1}$ was attained.

Taking into consideration the limited volume employed for the QIA methodology, some fluctuation throughout the experiment time could be observed (especially in Fig. 3). In fact, if the medium inside the Erlenmeyer is not completely homogenous at the sampling time, some samples may present larger biomass contents 
than others (thus contributing to some of the observed fluctuation in $\mathrm{TH} / \mathrm{Vol}$ ). However this effect will be the same in both the determined total hyphae length and total yeast area and, thus, be minimized regarding the TH/TY parameter (shown in Fig. 4). In fact, a much lesser fluctuation is observed in Fig. 4, confirming the ability of the QIA procedure to monitor the yeast-to-hyphae dimorphic change.

Concluding, Fig. 3 shows that for both strains, the presence of glucose as carbon source leads to an increase in the ability of $Y$. lipolytica to form hyphae, indicating the high specificity of this sugar in the induction of hyphae formation. Comparing the capability of both strains for dimorphic transition, MTLY40-2P strain was found to produce higher amounts of hyphae under similar conditions, except for the $\mathrm{CO}$ medium.

Thus, it could be established by the QIA morphological characterization that hydrophobic carbon sources should be preferred for Y. lipolytica W29 and MTLY40-2P growth when the yeast morphotype is required. On the other hand, hydrophilic carbon sources, as glucose and GlcNAc, in controlled pH conditions, caused a dimorphic transition towards the hyphae morphotype in Y. lipolytica W29 and MTLY40-2P strains.

Finally, it should be stressed that the developed QIA methodology allowed the quantification and characterization of $Y$. lipolytica cells into different morphotypes, ranging from yeast (oval) single cells to (filamentous) hyphae cells, automatically identifying the size and morphology of both morphotypes and further quantifying the proportion of each morphotype.

\subsection{Biotransformation experiments}

In industrial fermentations, the cells morphology is strongly affected by the rheological properties of the medium, oxygen transfer rate and nutrients consumption rate (Walker, 1998). Furthermore, the cells behavior in fermentations is greatly affected by their morphology. Hence, the control and understanding of cell morphology in the bioreactor is of key importance and QIA techniques can allow the identification of the best culture conditions for industrial processes implementation.

Experiments for $\gamma$-decalactone production with MTLY40-2P strain in two distinct morphotypes (yeast single cells and hyphae cells) were, therefore, performed. Regarding the hyphae morphotype, before the biotransformation experiments a pre-induction step for dimorphic transition of MTLY40-2P strain cells was performed, using glucose as carbon source, in order to insure that the cells were mainly in the hyphae morphotype. The $\gamma$-decalactone productivity of the hyphae morphotype cells was then compared with the yeast single cells morphotype (Fig. 5).

The kinetic profile of $\gamma$-decalactone production was similar for both tested morphotypes: an initial lag phase of around $72 \mathrm{~h}$ was apparent, followed by an increase in the aroma production, attaining a maximum concentration plateau and exhibiting no significant decrease from then until the end of the experiment. This is a typical profile for this strain in batch cultures (Braga et al., 2015b; Groguenin et al., 2004). Nevertheless, according to the cell morphotype a different behavior was observed in the aroma production, with the cells in the yeast morphotype exhibiting higher $\gamma$-decalactone production than the hyphae morphotype (c.a. 1.5fold). A similar production rate was observed during the first $168 \mathrm{~h}$, but from then onwards a higher aroma concentration was obtained for the cells in the yeast morphotype. During the biotransformation experiments, the overall growth was relatively slow with cell concentration only slightly increasing throughout the experiment time (ranging from $3.2 \times 10^{8}$ to $1.2 \times 10^{9}$ cells $\mathrm{mL}^{-1}$ ). Regarding microscopy examination, no significant morphological changes were observed during the course of the biotransformation experiments for each morphotype (Fig. 6). Furthermore, the QIA methodology allowed establishing a TH/TY ratio of $1370( \pm 816)$ $\mathrm{mm}^{-1}$ for the hyphae morphotype and of $8( \pm 7) \mathrm{mm}^{-1}$ for the yeast morphotype, confirming that no significant morphological changes were observed during the course of the biotransformation experiments. Also, in both conditions, the medium $\mathrm{pH}$ increased from 5.5 to 7.5 , at around $90 \mathrm{~h}$, decreasing again to 5.5 at the experiment end and the substrate (castor oil) was largely consumed, to a final concentration below $3 \mathrm{~g} \mathrm{~L}^{-1}$.

In fact, this work allowed to validate the adequacy and importance of cell morphology monitoring throughout dimorphic transition and biotransformation experiments, since differences in process productivity can be observed, as here demonstrated in $\gamma$ decalactone production by MTLY40-2P Y. lipolytica strain.

In conclusion, the use of QIA tools can be very helpful for cells morphological characterization and evaluation, during fermentation in industrial bioreactors, where cells are exposed to stress conditions, like pressure, oxygen limitations, mechanical shearing, that can lead to morphological alterations. Nevertheless, it is important to stress out that the cell morphological transition is strain dependent, thus being essential to study such behavior for each $Y$. lipolytica strain. Through microscopy and QIA techniques, a better understanding of cellular adaptation mechanisms can be achieved, and operational conditions adapted in order to reduce its impact in cells behavior.

\section{Acknowledgements}

The authors thank the Portuguese Foundation for Science and Technology (FCT) and the European Community fund FEDER, through Program COMPETE, under the scope of the Project RECI/BBB-EBI/0179/2012 (FCOMP-01-0124-FEDER-027462).

The authors also thank theFCT for the PhD grant SFRH/BD/63701/2009 to Adelaide Braga and post-doctoral grant SFRH/BPD/82558/2011 to Daniela Mesquita, and the strategic funding of ID/BIO/04469/2013 unit.

\section{References}

Barth, G., Gaillardin, C., 1997. Physiology and genetics of the dimorphic fungus Yarrowia lipolytica. FEMS Microbiol. Rev. 9, 219-237.

Bellou, S., Makri, A., Triantaphyllidou, I.-E., Papanikolaou, S., Aggelis, G., 2014. Morphological and metabolic shifts of Yarrowia lipolytica induced by alteration of the dissolved oxygen concentration in the growth environment. Microbiol. SGM 160, 807-817.

Braga, A., 2014. Strategies for increasing aroma production from castor oil by Yarrowia lipolytica. In: PhD Thesis. University of Minho, Braga, Portugal.

Braga, A., Mesquita, D.P., Amaral, A.L., Ferreira, E.C., Belo, I., 2015a. Aroma production by Yarrowia lipolytica in airlift and STR bioreactors: differences in yeast metabolism and morphology. Biochem. Eng. J. 93, 55-62.

Braga, A., Crutz-Le Coq, A.M., Dulermo, R., Nicaud, J.M., Belo, I., 2015b. Effect of POX genotype and Lip2p overexpression on lactone production and reconsumption during biotransformation of castor oil by Yarrowia lipolytica. Process. Biochem., http://dx.doi.org/10.1016/j.procbio.2015.05.019.

Cervantes-Chávez, J.A., Kronberg, F., Passeron, S., Ruiz-Herrera, J., 2009. Regulatory role of the PKA pathway in dimorphism and mating in Yarrowia lipolytica. Fungal Genet. Biol. 46, 390-399.

Coelho, M.A. Amaral, P.F.F. Belo, I, 2010. Yarrowia lipolytica: an industrial workhorse. In: Méndez-Vilas, A. (Ed.), Current Research, Technology and Education Topics in Applied Microbiology and Microbial Biotechnology. Formatex, pp. 930-944.

Coelho, M.A.Z., Coutinho, J.A.P., Ferreira, E.C., Mota, M., Belo, I., 2007. Analysis of the effects of hyperbaric gases on $S$. cerevisiae cell cycle through a morphological approach. Process. Biochem. 42, 1378-1383.

Coelho, M.A.Z., Belo, I., Pinheiro, R., Amaral, A.L., Mota, M., Coutinho, J.A.P., Ferreira, E.C., 2004. Effect of hyperbaric stress on yeast morphology: study by automated image analysis. Appl. Microbiol. Biotechnol. 66, 318-324.

Cox, P.W., Paul, G.C., Thomas, C.R., 1998. Image analysis of the morphology of filamentous microorganisms. Microbiology 144, 817-827.

Domínguez, A., Ferminãin, E., Gaillardin, C., 2000. Yarrowia lipolytica: an organism amenable to genetic manipulation as a model for analyzing dimorphism in fungi. In: Ernst, J.F., Schmidt, A. (Eds.), Dimorphism Human Pathogenic and Apathogenic Yeast. Karger, Basel, pp. 151-172.

Groguenin, A., Waché, Y., García, E.E., Aguedo, M., Husson, F., LeDall, M.T., Nicaud, J.-M., Belin, J.-M., 2004. Genetic engineering of the $\beta$-oxidation pathway in the 
yeast Yarrowia lipolytica to increase the production of aroma compounds. J. Mol. Catal. B Enzym. 28, 75-79.

Guevara-Olvera, L., Calvo-Mendez, C., Ruiz-Herrera, J., 1993. The role of polyamine metabolism in dimorphism of Yarrowia lipolytica. J. Gen. Microbiol. 139, 485-493.

Hurtado, C.A., Rachubinski, R.A., 1999. MHY1 encodes a C2H2-type zinc finger protein that promotes dimorphic transition in the yeast Yarrowia lipolytica. J. Bacteriol. 181, 3051-3057.

Kawasse, F.M., Amaral, P.F., Rocha-Leão, M.H.M., Amaral, A.L., Ferreira, E.C., Coelho, M.A.Z., 2003. Morphological analysis of Yarrowia lipolytica under stress conditions through image processing. Bioprocess. Biosyst. Eng. 25, 371-375.

Kim, J., Cheon, S.A., Park, S., Song, Y., Kim, J.Y., 2000. Serum-induced hyphae formation in the dimorphic yeast Yarrowia lipolytica. FEMS Microbiol. Lett. 190, 9-12.

Martinez-Vazquez, A., Gonzalez-Hernandez, A., Domínguez, A., Rachubinski, R., Riquelme, M., Cuellar-Mata, P., Guzman, J.C.T., 2013. Identification of the transcription factor Znc1p, which regulates the yeast-to-hypha transition in the dimorphic yeast Yarrowia lipolytica. PLos One 8, e66790.

Morales-Vargas, A.T., Domínguez, A., Ruiz-Herrera, J., 2012. Identification of dimorphism-involved genes of Yarrowia lipolytica by means of microarray analysis. Res. Microbiol. 163, 378-387.

Novotony, C., Dolezalova, L., Lieblova, J., 1994. Dimorphic growth and lipase production in lipolytic yeasts-Yarrowia lipolytica, Candida rugosa, Torulopsis erbinii, Candida curvata, and Candida guilliermondii. Folia Microbiol. 39, $71-73$.
Ofek, I., Whitnack, E., Beachey, E.H., 1983. Hydrophobic interactions of group A streptococci with hexadecane droplets. J. Bacteriol. 154, 139-145.

Ota, Y., Oikawa, S., Morimoto, Y., Minoda, Y., 1984. Nutritional factors causing mycelial development of Saccharomycopsis lipolytica. Agric. Biol. Chem. 48 1933-1939.

Papanikolaou, S., Muniglia, L., Chevalot, I., Aggelis, G., Marc, I., 2002. Yarrowia lipolytica as a potential producer of citric acid from raw glycerol. J. Appl. Microbiol. 92, 737-744

Perez-Campo, F.M., Dominguez, A., 2001. Factors affecting the morphogenetic switch in Yarrowia lipolytica. Curr. Microbiol. 43, 429-433.

Pons, M.N., Vivier, H., 1998. Beyond filamentous species. Adv. Biochem. Eng. Biotechnol. 60, 61-93.

Pons, M.N., Vivier, H., Rémy, J.F., Dodds, J.A., 1993. Morphological characterization of yeast by image analysis. Biotechnol. Bioeng. 40, 187-193.

Puthli, M.S., Rathod, V.K., Pandit, A.B., 2006. Enzymatic hydrolysis of castor oil: process intensification studies. Biochem. Eng. J. 31, 31-41.

Rodríguez, C., Domínguez, A., 1984. The growth characteristics of Saccharomycopsis lipolytica: morphology and induction of mycelial formation. Can. J. Microbiol. 30, 605-612.

Ruiz-Herrera, J., Sentandreu, R., 2002. Different effectors of dimorphism in Yarrowia lipolytica. Arch. Microbiol. 178, 477-483.

Szabo, R., Stofanikova, V., 2002. Presence of organic sources of nitrogen is critical for filament formation and $\mathrm{pH}$-dependent morphogenesis in Yarrowia lipolytica. FEMS Microbiol. Lett. 206, 45-50.

Zinjarde, S., Pant, A., Deshpande, M., 1998. Dimorphic transition in Yarrowia lipolytica isolated from oil-polluted sea water. Mycol. Res. 102, 553-558. 